\title{
Successful Removal of an Entrapped Intravascular Ultrasound Catheter Using a 0.010-inch Guidewire and Compatible Balloon Catheter System
}

\author{
Hirokazu Yokoi, Yutaro Ota, Tsubasa Komai, Shinya Yamazaki, \\ Takashi Yanagiuchi, Shunpei Ushimaru, and Taku Kato
}

\begin{abstract}
Intravascular ultrasound (IVUS) catheter is a user-friendly imaging device widely used during percutaneous coronary intervention (PCl). However, IVUS catheter entrapment is an infrequent but serious complication associated with PCl. Case 1 was an 87-year-old woman on hemodialysis who had non-ST elevation myocardial infarction with total occlusion in the middle left anterior descending artery (LAD). PCI was performed with two drug-eluting stents (DESs) under IVUS guidance, but the IVUS catheter was entrapped at the implanted stent, leading to stent deformation. Case 2 was an 86-year-old woman on hemodialysis who had stable angina with severe calcified stenosis in the middle LAD. IVUS-guided rotational atherectomy was performed, but the IVUS catheter was stuck in the implanted DES with stent deformation. In both patients, we attempted to use a 0.010inch guidewire and compatible balloon catheter system that can pass through a $6-\mathrm{Fr}$ guide catheter simultaneously with the IVUS catheter, and the entrapped IVUS catheters were successfully removed from the implanted stents. This retrieval method is very convenient to apply during bailout and should be recommended especially in $\mathrm{PCl}$ using a 6 - $\mathrm{Fr}$ guide catheter.
\end{abstract}

KEY WORDS: complications, drug-eluting stent, intravascular ultrasound catheter entrapment, percutaneous coronary intervention, 0.010 -inch guidewire and compatible balloon catheter system

\section{Introduction}

Intravascular ultrasound (IVUS) imaging is a user-friendly technique widely used during percutaneous coronary intervention (PCI). However, the entrapment of an IVUS catheter is a rare but serious complication associated with $\mathrm{PCI}^{1)}$. Although an entrapped IVUS catheter could be removed using a variety of retrieval techniques ${ }^{2-4)}$, there are few convenient troubleshooting methods in PCI with the commonly used 6-Fr guide catheter.

In contrast, a 0.010-inch guidewire-compatible balloon catheter (IKAZUCHI X Hyper, Kaneka, Japan) has been designed exclusively for a 0.010-inch guidewire ; the diameter of the catheter was made smaller than those of other balloon catheters compatible with 0.014-inch guidewires. The effectiveness of the 0.010-inch guidewire system has been demonstrated in several clinical situations requiring a reduced guide catheter size ${ }^{5,6)}$. Therefore, a 0.010-inch guidewire and compatible balloon cathe-

Department of Cardiology, Rakuwakai Otowa Hospital, 2 Otowa-chinjicho, Yamashina-ku, Kyoto, 607-8062, Japan

E-mail: h-yokoi@koto.kpu-m.ac.jp

Received: July 20, 2020; Accepted: January 14, 2021

doi: 10.7793/jcad.27.20-00019

(C)The Japanese Coronary Association ter system can pass in parallel with an IVUS catheter into a 6-Fr guide catheter. Here, we report the cases of two patients in whom a 0.010-inch guidewire system was successfully used to remove the entrapped IVUS catheter from the implanted stent.

\section{Case 1}

An 87-year-old woman with end-stage renal disease (ESRD) on hemodialysis was transferred to our hospital due to non-STsegment elevation myocardial infarction. She had a history of stent implantation in the proximal left anterior descending artery (LAD). Urgent coronary angiography (CAG) revealed total occlusion in the middle LAD (Fig. 1A). Primary PCI was performed using a 6-Fr guide catheter (Hyperion SPB3.5, Asahi Intecc, Japan) via the right femoral artery. After the culprit lesion was dilated using a semi-compliant balloon catheter (CoreThrough Ace, $2.0 \times 15$ mm, Fukuda Denshi, Japan) (Fig. 1B), Thrombolysis in Myocardial Infarction (TIMI) grade 3 flow was restored (Fig. 1C). An IVUS catheter (OptiCross, Boston Scientific, USA) could not be advanced into the distal tortuous LAD ; nevertheless, IVUS pullback imaging was performed from the middle to the proximal LAD. It revealed the presence of a stent with good expansion, which was previously implanted in the proximal $\mathrm{LAD}$, and a moderate calcified plaque in the middle LAD (Fig. 1C). Two overlapping everolimus-eluting stents 

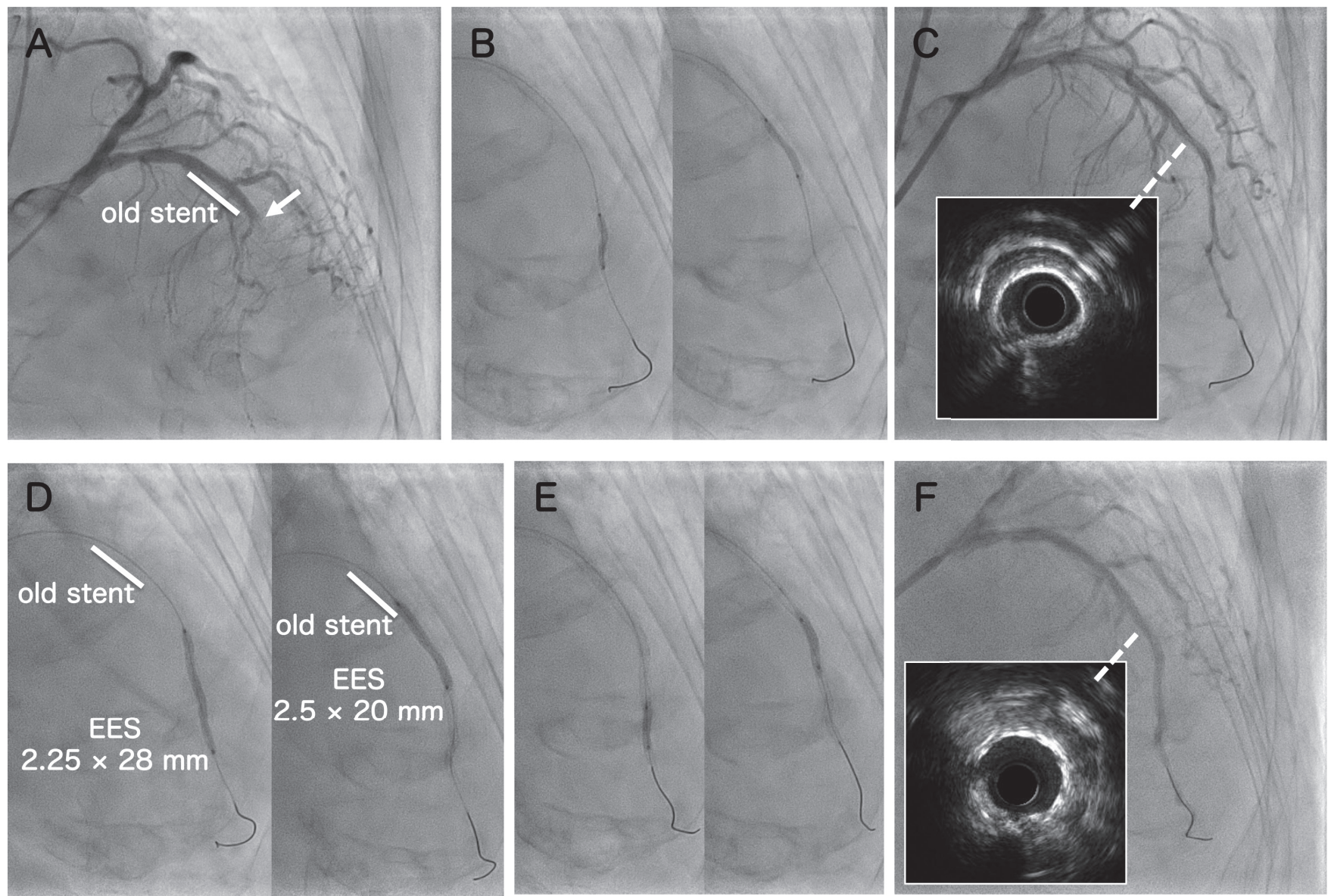

Fig. 1 Emergency CAG and primary PCI for NSTEMI in Case 1.

A: Left CAG revealed a patent old stent in the proximal LAD and total occlusion in the middle LAD (white arrow) (right anterior cranial view).

B: Pre-dilation with a semi-compliant balloon catheter.

C: Angiography and IVUS image showed a calcified lesion in the middle LAD and a tortuous lesion in the distal LAD.

D: Two overlapping everolimus-eluting stents (EESs) were deployed from the middle to the distal LAD distally with an overlap with the old stent.

E: Post-dilation with a non-compliant balloon catheter.

F: Angiography and IVUS image post-stent dilation showed satisfactory stent apposition and expansion in the calcified lesion.

(EESs) (Synergy, $2.5 \times 20 \mathrm{~mm}, 2.25 \times 28 \mathrm{~mm}$, Boston Scientific) were deployed distally with minimal overlap with the old stent in the proximal LAD, completely covering the diffuse plaque (Fig. 1D). The stents were subsequently post-dilated with a non-compliant balloon catheter (Hiryu Plus, $2.25 \times 12 \mathrm{~mm}$, Terumo, Japan) (Fig. 1E). The IVUS image showed relatively good stent apposition and expansion in the calcified lesion (Fig. $1 \mathrm{~F})$; however, the IVUS catheter was trapped at the distal stent strut, and the distal portion of the stent appeared to be deformed on fluoroscopy (Fig. 2A). In order to rescue the situation, the entrapped IVUS catheter could be pushed forward, but could not be pulled back. Although we cut the proximal IVUS shaft and inserted a guide extension catheter (GuideLiner, 6 Fr, Teleflex, USA) over the IVUS shaft, the guide extension catheter was unable to advance beyond the previous stent in the proximal LAD. Then, after the IVUS imaging core was removed, a 0.021-inch guidewire was inserted into the tube, and the entrapped IVUS catheter was pushed and rotated. But the IVUS catheter could not be removed. Next, we carefully passed a 0.014-inch tapered guidewire (XT-R, Asahi Intecc) through the crushed stent, using the double-guide catheter technique via the left femoral artery; however, the microcatheter (Corsair Pro, Asahi Intecc) and the 0.014-inch guidewire-compatible small balloon catheter (Sapphire II Pro, $1.0 \times 5 \mathrm{~mm}$, OrbusNeich, Hong Kong) were unable to traverse through the stent (Fig. 2B). Finally, we cautiously passed a 0.010-inch guidewire (Athlete Eel Slender, Japan Lifeline, Japan) through the stent (Fig. 2C). After a 0.010-inch guidewire-compatible balloon catheter (IKAZUCHI X Hyper, $1.5 \times 9$ $\mathrm{mm}$, Kaneka) was successfully passed through the crushed stent, the IVUS catheter was retrieved conveniently (Fig. 2D). The deformed distal stent was re-dilated with a non-compliant balloon (Hiryu Plus, $2.25 \times 12 \mathrm{~mm}$ ) (Fig. 2E), and the proximal stent was post-dilated using another non-compliant balloon (Hiryu Plus, $3.0 \times 8 \mathrm{~mm}$, Terumo). Final angiography revealed satisfac- 

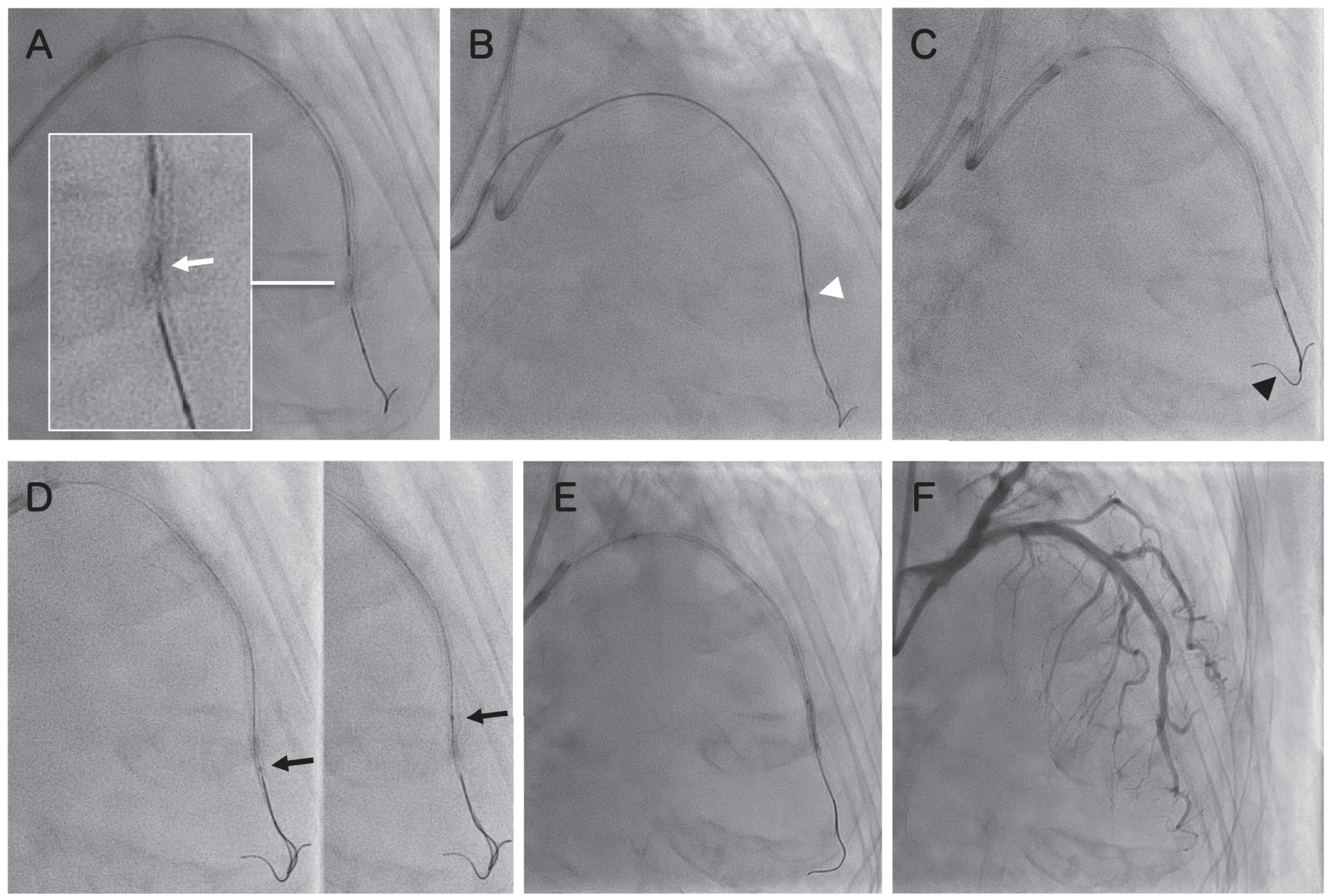

Fig. 2 Retrieval of an entrapped IVUS catheter in Case 1.

A: The IVUS catheter was stuck in the distal stent strut with stent crush (white arrow).

B: A microcatheter (white arrow head) on an XT-R guidewire could not pass through the crushed stent using the double-guide catheter technique.

C: A 0.010-inch guidewire and compatible balloon catheter system (black arrow head) was able to cross the deformed stent.

D: The entrapped IVUS catheter (black arrow) was successfully removed.

E: Re-dilation of the crushed stent with a non-compliant balloon.

F: Final CAG after PCI.

tory stent expansion without residual stenosis and TIMI grade 3 flow in the LAD (Fig. 2F).

\section{Case 2}

An 86-year-old woman with ESRD on hemodialysis was admitted to our hospital due to stable angina. CAG and IVUS (AltaView, Terumo) findings revealed severe calcified stenosis in the middle LAD (Fig. 3A). Rotational atherectomy with a $1.5-\mathrm{mm}$ burr (RotaLink Plus, Boston Scientific) was performed using a 6-Fr guide catheter (Hyperion SPB3.5, Asahi Intecc) (Fig. 3B). After dilation using a cutting balloon (Wolverine, $2.5 \times 10 \mathrm{~mm}$, Boston Scientific) (Fig. 3C), a sirolimus-eluting stent (SES) (Orsiro, $3.0 \times 40 \mathrm{~mm}$, Biotronik, Switzerland) was implanted to fully cover the tandem plaques (Fig. 3D). The IVUS image after post-dilation with a non-compliant balloon (NC Kamui, $3.5 \times 12$ $\mathrm{mm}$, Asahi Intecc) showed adequate stent apposition and expansion (Fig. 3E, F) ; however, the IVUS catheter was stuck at the distal stent strut, and the distal edge of the stent appeared to be deformed on fluoroscopy (Fig. 4A). In order to rescue the situation, the entrapped IVUS catheter could be pushed forward, but could not be pulled back. Then, after the IVUS imaging core was removed, a 0.021-inch guidewire was inserted into the tube, and the entrapped IVUS catheter was pushed and rotated. But the IVUS catheter could not be removed. Therefore, we attempted to use a 0.010 -inch guidewire system that can be inserted in parallel with the IVUS catheter through a 6-Fr guide catheter, referring to the previous case. A 0.010-inch Athlete Eel Slender guidewire and a 1.5-mm IKAZUCHI X Hyper balloon catheter were successfully passed through the crushed stent (Fig. 4B). After the deformed stent was re-dilated with the 0.010-inch compatible balloon (Fig. 4C), the IVUS catheter was retrieved successfully (Fig. 4D). The stent was post-dilated by another non-compliant balloon catheter (Tasuki, $2.5 \times 12 \mathrm{~mm}$, Kaneka) (Fig. 4E). Final angiography showed good stent expansion with- 

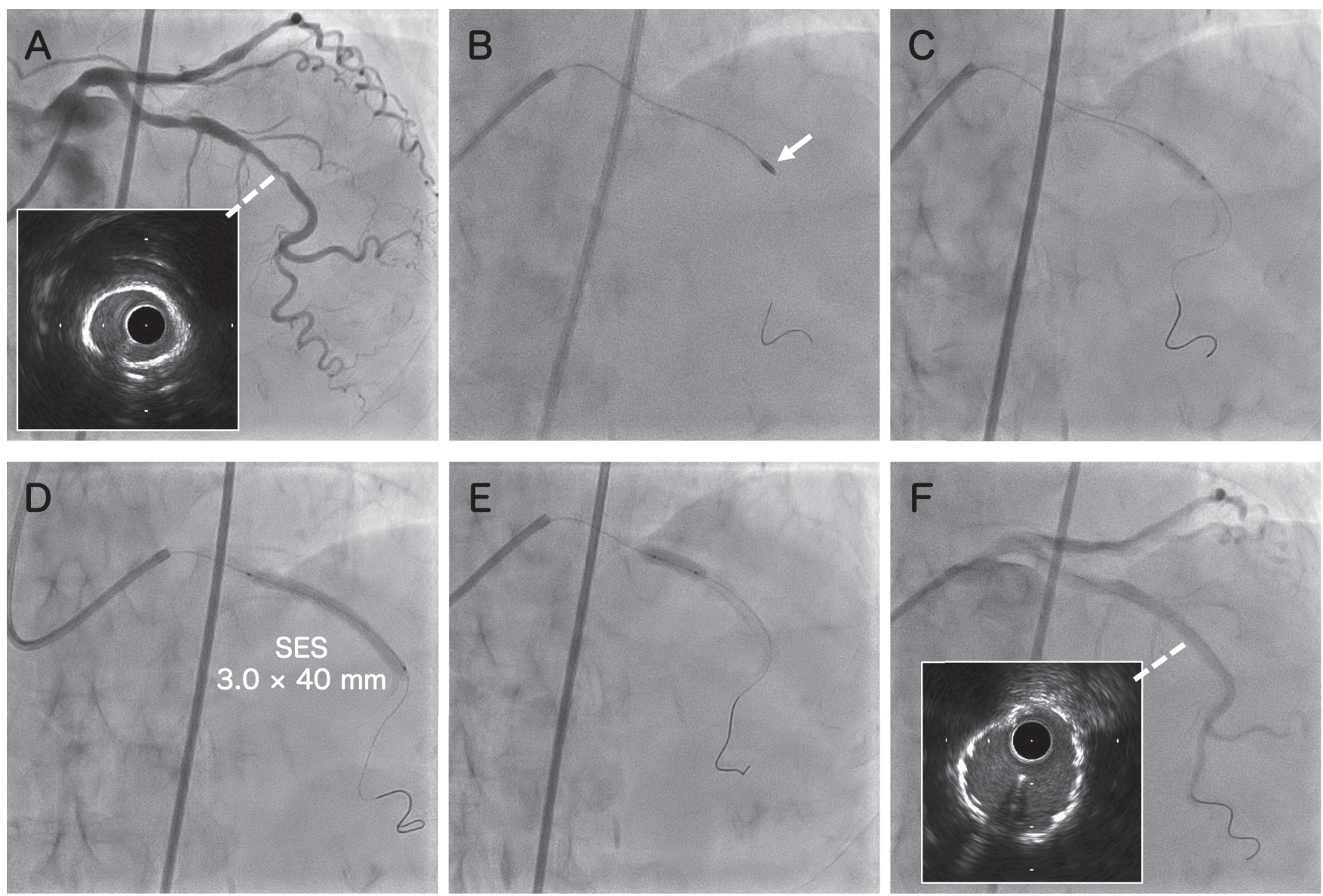

Fig. 3 Elective PCI for stable angina in Case 2.

A: Left CAG and IVUS image before PCI revealed a severe calcified lesion in the middle LAD (right anterior cranial view).

B: Rotational atherectomy with a 1.5 -mm burr (white arrow).

C: Dilation with a cutting balloon catheter.

D: Implantation of a sirolimus-eluting stent (SES) from the proximal to the middle LAD.

E: Post-dilation with a non-compliant balloon catheter.

F: Angiography and IVUS image post-stent dilation showed good stent apposition and expansion in the calcified lesion.

out residual stenosis (Fig. 4F).

\section{Discussion}

IVUS is an invasive imaging technique used to visualize coronary cross-sectional anatomy and is superior to CAG in assessing vessel size, lesion severity, plaque volume, and calcium content $^{7,8)}$. It also provides complementary procedural information on lesions requiring PCI when determining adequate stent sizing and confirming optimal stent deployment in real time ${ }^{9)}$. Moreover, IVUS can assess stent underexpansion, malapposition, and edge dissection after stent implantation, which may lead to stent thrombosis and restenosis ${ }^{10,11)}$. With the advent of drug-eluting stents (DESs), IVUS-guided DES implantation has been associated with a significant reduction in target vessel revascularization, cardiac death, and stent thrombosis compared with angiography-guided implantation ${ }^{12)}$.

However, the entrapment of an IVUS catheter is an infrequent but serious complication associated with PCI, and a surgical procedure may be required to retrieve the catheter ${ }^{1)}$. The IVUS catheters used in our patients, an OptiCross IVUS and an AltaView IVUS, incorporated a single 40- and 60-MHz transducer within a 3.2- and 3.0-Fr short monorail system, respectively. When advancing the catheter through a stented vessel, catheters that do not completely encapsulate the guidewire may engage the stent between the junction of the catheter and guidewire, resulting in catheter/guidewire entrapment, catheter tip separation, and/or stent dislocation. The primary potential mechanism of IVUS catheter entrapment is that the guidewire exit port of the IVUS catheter gets caught at the stent strut when the IVUS catheter is pulled back after stent implantation ${ }^{13,14)}$. Previous reports demonstrated that the IVUS catheter was almost stuck in tortuous and/ or calcified lesions ${ }^{15,16)}$. Similarly, our cases also suggest that tortuous vessels and calcified lesions would be a high-risk factor of IVUS catheter entrapment. In such cases, a special attention 

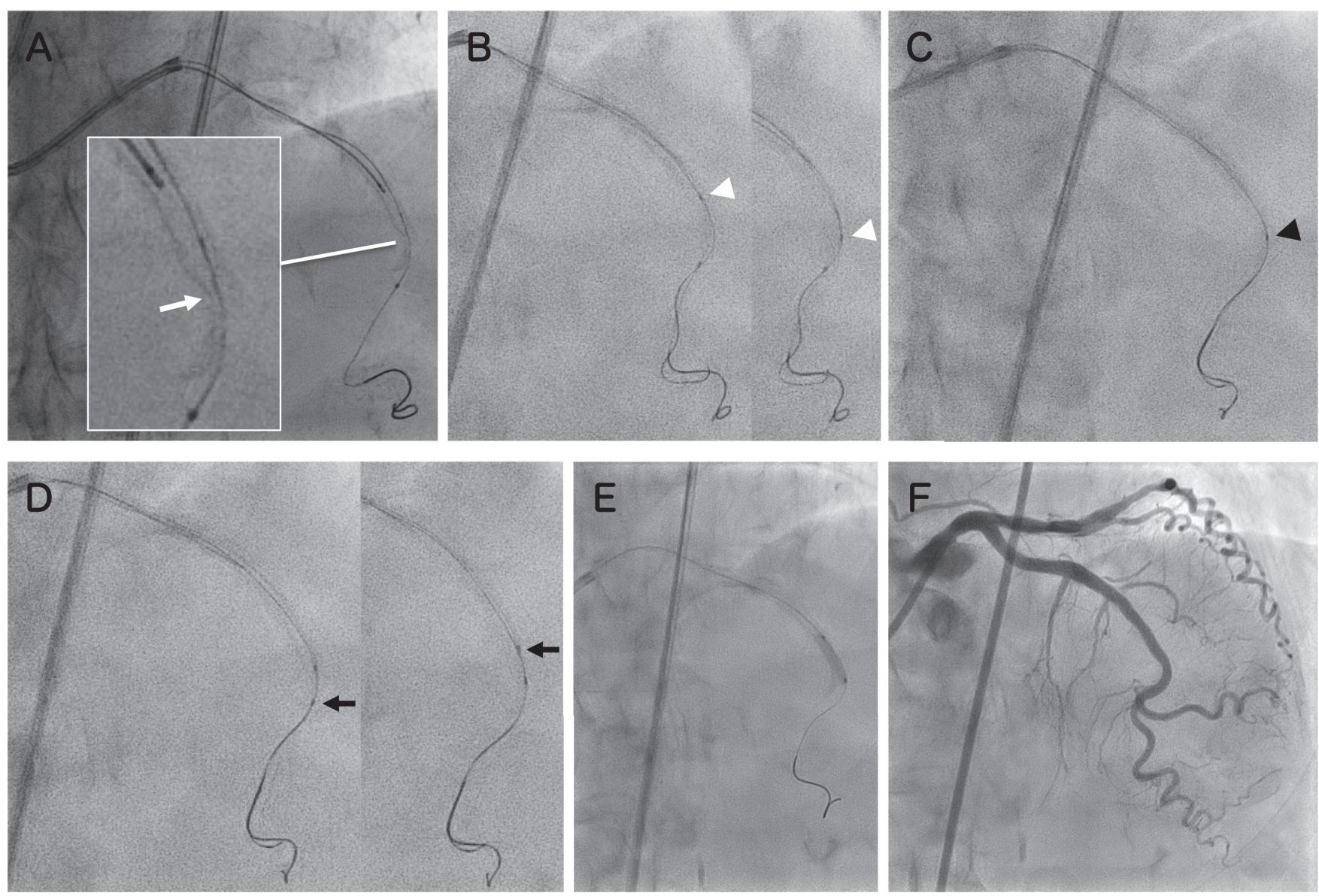

Fig. 4 Retrieval of an entrapped IVUS catheter in Case 2.

A: The IVUS catheter was stuck in the distal stent edge with stent deformation (white arrow).

B: A 0.010-inch guidewire and a 0.010-inch compatible balloon catheter (white arrow head) were able to cross the deformed stent through a 6-Fr guide catheter.

C: Dilation of the deformed stent with a 0.010-inch compatible balloon catheter (black arrow head).

D: The entrapped IVUS catheter (black arrow) was successfully removed.

E: Re-dilation of the deformed stent with a non-compliant balloon.

F: Final CAG after PCI.

should be paid to the removal of the IVUS catheter as follows Before the removal of the IVUS catheter, it is important to return the imaging core to the original position in a live mode. Operators should then carefully remove the IVUS catheter under fluoroscopy. Nevertheless, operators should stop the removal procedure if they feel any resistance.

Several percutaneous bailout techniques have been reported, including the rotation technique, buddy wire technique, double-guide catheter technique, covering the exit port of an IVUS catheter with a balloon catheter on the guidewire with which the IVUS catheter is delivered, replacing the IVUS imaging core with a larger diameter (OptiCross, 0.025 inch or AltaView, 0.021 inch) guidewire, and covering the IVUS catheter with a guide catheter extension system ${ }^{2-4)}$. The double-guide catheter technique requires a different access route. Covering the exit port of an IVUS catheter with a balloon catheter is considered during
PCI with a 7-Fr or 8-Fr guide catheter. The imaging core of an AltaView IVUS can be removed without cutting the shaft, but the imaging core of an OptiCross IVUS cannot be removed without cutting the shaft. Moreover, the shaft of both IVUS catheters must be cut to be covered by a guide extension catheter. Finally, there is a method to pull the guidewire with which the IVUS catheter is delivered and separate the guidewire from the IVUS catheter; however, it may be difficult to re-cross the guidewire through the crushed stent. The retrieval methods are limited especially during PCI using the well-known 6-Fr guide catheter.

In contrast, a 0.010-inch guidewire and compatible balloon catheter system is considered as a key component of the "slender system" that was first introduced in Japan, and it is mostly used for 5-Fr transradial PCI. It has been shown to be useful for treating bifurcation lesions when there is a need to cross a stent-jailed side branch and when simultaneous kissing balloon inflation is 
A

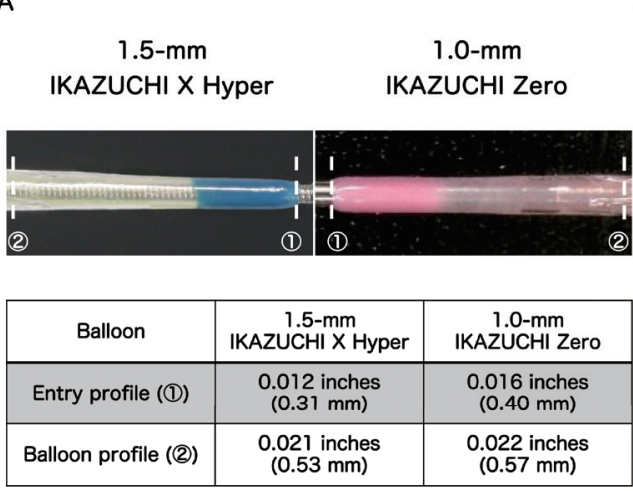

B

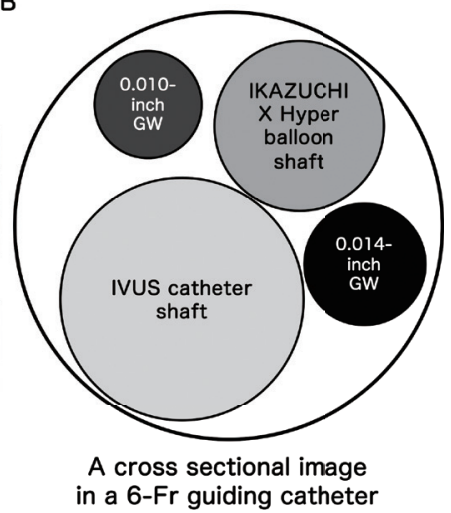

Fig. 5 Very low profile of an IKAZUCHI X Hyper balloon catheter.

A: Visual comparison of the entry profile between a 0.010-inch compatible 1.5-mm IKAZUCHI X Hyper balloon catheter and a 0.014-inch compatible 1.0-mm balloon catheter (IKAZUCHI Zero, Kaneka). The outer diameter of the entry tip of a 1.5-mm IKAZUCHI $\mathrm{X}$ Hyper balloon is smaller than the outer diameter of a conventional 0.014-inch guidewire. B: A cross sectional image in a 6-Fr guiding catheter. A standard 0.014-inch guidewire and a 0.014-inch compatible balloon catheter cannot pass through a 6-Fr guide catheter in parallel with an IVUS catheter, but a 0.010-inch guidewire and an IKAZUCHI X Hyper balloon catheter can pass through simultaneously with an IVUS catheter. IVUS: intravascular ultrasound, GW: guidewire

performed in a 5 -Fr guide catheter ${ }^{5,6)}$. The 0.010 -inch guidewire-compatible balloon catheter (IKAZUCHI X Hyper) has a very low profile in terms of the balloon tip and catheter shaft. The outer diameter of the entry profile of the $1.5-\mathrm{mm}$ IKAZUCHI X Hyper balloon catheter is 0.012 inches, which is smaller than the outer diameter of the standard 0.014-inch guidewire (Fig. 5A). The very low entry profile of the balloon enables it to cross microchannels in chronic total occlusions, bifurcation, and tortuous/bent lesions ${ }^{17,18)}$. In addition, the distal shaft outer diameter of the 1.5-mm IKAZUCHI X Hyper balloon catheter is smaller $(2.1 \mathrm{Fr})$ than that of a 0.014-inch compatible balloon catheter. Although a conventional guidewire and balloon catheter cannot pass generally in parallel with an IVUS catheter through a 6-Fr guide catheter, a 0.010-inch guidewire and a $1.5-\mathrm{mm}$ IKAZUCHI X Hyper balloon catheter can be used simultaneously with an OptiCross IVUS catheter (3.2 Fr) or an AltaView IVUS catheter (3.0 Fr) (Fig. 5B). Alternatively, a Refinity IVUS catheter (Philips, Nederland BV) is a commercially available short monorail type-IVUS catheter, and can be theoretically used in this system as the outer diameter of a Refinity IVUS catheter (3.1 Fr) is smaller than that of an OptiCross IVUS catheter.

This is the first report to describe two cases in which the usage of a 0.010-inch guidewire system was effective in the removal of the entrapped IVUS catheter from the implanted stent. The retrieval method is very convenient to apply during bailout without requiring for another access route, a 7-Fr or larger guide catheter, and IVUS catheter shaft cutting. It should be recommended when an IVUS catheter is entrapped at an implanted stent in a
6-Fr guide catheter.

\section{Conclusion}

A 0.010-inch guidewire and compatible balloon catheter system can pass through a 6-Fr guide catheter simultaneously with an IVUS catheter. The retrieval method using a 0.010 -inch guidewire system is very convenient to perform during bailout and should be recommended when an IVUS catheter is entrapped in an implanted stent with a 6-Fr guide catheter.

\section{Conflicts of interest}

The authors declare that they have no conflict of interest.

\section{References}

1) Morishima A, Yoshida Y, Heima D, et al: Successful surgical treatment of intravascular ultrasonography catheter entrapment. Gen Thorac Cardiovasc Surg 2011; 59: 609-611

2) Sasseen BM, Burke JA, Shah R, et al: Intravascular ultrasound catheter entrapment after coronary artery stenting. Catheter Cardiovasc Interv 2002; 57: 229-233

3) Kim JY, Lee NH, Cho YH, et al: Recanalization of an accidentally crushed coronary stent by intravascular ultrasonography catheter entrapment. Korean Circ J 2011; 41: 327-330

4) Ashikaga T, Inagaki $\mathrm{H}$, Satoh $\mathrm{Y}$, et al: Proximal balloon deflation technique: A novel method to retrieve retained or entrapped equipment from the coronary system. Cardiovasc Revasc Med 2012; 13: 253-255

5) Yoshimachi F, Masutani M, Matsukage T, et al: Kissing balloon technique within a $5 \mathrm{Fr}$ guiding catheter using 0.010 inch guidewires and 0.010 inch guidewire-compatible balloons. J Invasive Cardiol 2007; 
19: $519-524$

6) Otsuka Y, Kokubu N, Kasahara Y: Usefulness of a 0.010-inch guidewire compatible balloon for crossing stent-jailed sidebranches. Int $\mathrm{J}$ Cardiol 2011; 148: 228-231

7) Harrison RW, Aggarwal A, Ou FS, et al: Incidence and outcomes of no-reflow phenomenon during percutaneous coronary intervention among patients with acute myocardial infarction. Am J Cardiol 2013; 111: $178-184$

8) Roy P, Steinberg DH, Sushinsky SJ, et al: The potential clinical utility of intravascular ultrasound guidance in patients undergoing percutaneous coronary intervention with drug-eluting stents. Eur Heart J 2008; 29: 1851-1857

9) Morino Y, Tamiya S, Masuda N, et al: Intravascular ultrasound criteria for determination of optimal longitudinal positioning of sirolimus-eluting stents. Circ J 2010; 74: 1609-1616

10) Kang SJ, Ahn JM, Song H, et al: Comprehensive intravascular ultrasound assessment of stent area and its impact on restenosis and adverse cardiac events in 403 patients with unprotected left main disease. Circ Cardiovasc Interv 2011; 4: 562-569

11) Cook $S$, Wenaweser $P$, Togni $M$, et al: Incomplete stent apposition and very late stent thrombosis after drug-eluting stent implantation. Circulation 2007; 115: 2426-2434

12) Elgendy IY, Mahmoud AN, Elgendy AY, et al: Outcomes with intra- vascular ultrasound-guided stent implantation: A meta-analysis of randomized trials in the era of drug-eluting stents. Circ Cardiovasc Interv 2016; 9: e003700

13) Shimahara Y, Kobayashi J, Nakajima H, et al: Surgical treatment of entrapped intravascular ultrasonography catheter. Gen Thorac Cardiovasc Surg 2012; 60: 112-114

14) Hamamoto M, Futagami D: Successful surgical removal of an entrapped intravascular ultrasonography catheter in the left circumflex coronary artery. Gen Thorac Cardiovasc Surg 2012; 60: 830-833

15) Hiraya D, Sato A, Hoshi T, et al: Incidence, retrieval methods, and outcomes of intravascular ultrasound catheter stuck within an implanted stent: Systematic literature review. J Cardiol 2020; 75: 164 170

16) Tsukui $T$, Sakakura $K$, Momomura $S$, et al: A novel method for prevention of intravascular ultrasound catheter entrapment using soft guide extension catheter. Cardiovasc Interv Ther 2020; 35: 405-406

17) Matsukage T, Yoshimachi F, Masutani M, et al: A new 0.010-inch guidewire and compatible balloon catheter system: the IKATEN registry. Catheter Cardiovasc Interv 2009; 73: 605-610

18) Matsukage T, Masutani M, Yoshimachi F, et al: A prospective multicenter registry of 0.010-inch guidewire and compatible system for chronic total occlusion: the PIKACHU registry. Catheter Cardiovasc Interv 2010; 75: 1006-1012 